\title{
Invariant differential equations and the Adler-Gel'fand-Dikii bracket
}

\author{
Artemio González-López and Rafael Hernández Heredero \\ Departamento de Física Teórica II, Facultad de Ciencias Físicas, Universidad \\ Complutense, 28040 Madrid, Spain \\ Gloria Marí Beffa \\ Department of Mathematics, University of Wisconsin, Madison, Wisconsin 53706
}

(Received 24 March 1997; accepted for publication 30 June 1997)

In this paper we find an explicit formula for the most general vector evolution of curves on $\mathbf{R P}^{n-1}$ invariant under the projective action of $\operatorname{SL}(n, \mathbf{R})$. When this formula is applied to the projectivization of solution curves of scalar Lax operators with periodic coefficients, one obtains a corresponding evolution in the space of such operators. We conjecture that the formula we have found gives another alternative definition of the second KdV Hamiltonian evolution under appropriate conditions. In other words, both evolutions are identical provided that the vector differential invariant characterizing the $\operatorname{SL}(n, \mathbf{R})$-invariant evolution on the space of projectivized curves is identified with the coefficients of the Hamiltonian pseudodifferential operator. We prove the above facts for $n \leqslant 6$, and further simplify both evolutions in appropriate coordinates so that one can attempt to prove the equivalence for arbitrary $n$. (C) 1997 American Institute of Physics.

[S0022-2488(97)02111-7]

\section{INTRODUCTION}

In an attempt to generalize the bi-Hamiltonian character of the Korteweg-deVries (KdV) equation, Adler ${ }^{1}$ defined a family of second Hamiltonian structures with respect to which the generalized higher-dimensional KdV equations could also be written as Hamiltonian systems. Jacobi's identity for these brackets was proved by Gel'fand and Dikii in Ref. 2. These Poisson structures are called second Hamiltonian KdV structures or Adler-Gel'fand-Dikii brackets. Since the original definition of Adler was quite complicated and not very intuitive, alternative definitions have been subsequently offered by several authors, most notably by Kupershmidt and Wilson in Ref. 3, and by Drinfel'd and Sokolov in Ref. 4. Once higher-dimensional KdV equations were proved to be bi-Hamiltonian, their integrability was established via the usual construction of a sequence of Hamiltonian structures with commuting Hamiltonian operators. The second Hamiltonian Structure in the hierarchy of KdV brackets coincides with the usual second Poisson bracket for the KdV equation, that is, the canonical Lie-Poisson bracket on the dual of the Virasoro algebra. This is the only instance in which the second $\mathrm{KdV}$ bracket is linear.

A subject apparently unrelated to the Hamiltonian structures of partial differential equations is the theory of Klein geometries and differential and geometric invariants. This theory had its high

point in the last century before the appearance of Cartan's approach to differential geometry, and it is closely related to equivalence problems. Namely, one poses the question of equivalence of two geometrical objects under the action of a certain group, that is, when can one of those objects be taken to the other one using a transformation belonging to the given group? For example, given two curves on the plane, when are they equivalent under an Euclidean motion? Or, when are they the same curve, up to parametrization?, etc. One answer can be given in terms of invariants, namely, expressions that depend on the objects under study and that do not change under the action of the group. If two objects are to be equivalent, they must have the same invariants. If these invariants are functions on some jet space (for example, if they depend on the curve and its 
derivatives with respect to the parameter), then they are called differential invariants. In the case of curves on the Euclidean plane under the action of the Euclidean group, the basic differential invariant is known to be the Euclidean curvature. Within the natural scope of the study of equivalence problems and their invariants lies also the description of invariant differential equations, symmetries, relative invariants, etc. For example, recently Olver et al. ${ }^{5}$ used these ideas to characterize all scalar evolution equations invariant under the action of a subgroup of the projective group in the plane, a problem of interest in the theory of image processing. See Olver's book ${ }^{6}$ for an account of the state of the subject.

In this paper we establish a connection between the foregoing two theories while trying to answer the following question. Let $L(t, \theta)$ be a family of scalar differential operators with periodic coefficients following an evolution (in $t$ ) which is Hamiltonian with respect to the AdlerGel'fand-Dikii bracket. Consider a family of solution curves $\xi(t, \theta)$ associated to $L(t, \theta)$. Is there a simple and explicit way to describe the evolution of $\xi(t, \theta)$ ? The importance of studying the space of solutions of $L$ was pointed out by Wilson in Ref. 7. Also in Ref. 8, from a different point of view than the one presented here, a description of this evolution was given and proven to be $\operatorname{SL}(n, \mathbf{R})$ invariant. These curves are also used to provide a discrete invariant of the Poisson bracket, one of the two invariants which classify the symplectic leaves. ${ }^{9}$ Here, we aim to show that the evolution of the solution curves is of relevance in itself, and can be described using the theory of differential invariants. We will see that the evolution of the projectivization $\phi(t, \theta)$ of a solution curve is invariant under the projective action of $\operatorname{SL}(n, \mathbf{R})$. Following Olver's approach, ${ }^{6}$ we will write explicitly the most general (vector) evolution of curves on real $(n-1)$-dimensional projective space $\mathbf{R} \mathbf{P}^{n-1}$ of the form

$$
\phi_{t}=F\left(\phi, \phi_{\theta}, \phi_{\theta \theta}, \ldots\right)
$$

which is invariant under the $\operatorname{SL}(n, \mathbf{R})$ projective action. Moreover, under certain conditions that we will state precisely in the paper, we conjecture that every $\operatorname{SL}(n, \mathbf{R})$-invariant evolution of curves on $\mathbf{R P}^{n-1}$ must correspond to an Adler-Gel'fand-Dikii Hamiltonian evolution in the space of time-dependent $n$th order scalar differential operators with periodic coefficients. This correspondence, which provides an alternative definition of the Adler-Gel'fand-Dikii bracket, will be described in detail and shown to be true for many fixed values of $n$. Unfortunately, we haven't succeeded in proving the general case, which is considerably more involved. We will nevertheless guide the reader in simplifying the proof in the general case, so that he or she can attempt to prove the conjecture for any particular value of $n$.

\section{NOTATION AND BASIC FACTS}

In this Section we will set the notation used in the rest of the paper, and recall some elementary properties of the projectivized solution curves of scalar Lax operators. Denote by $\mathscr{b}_{n}$ the infinite-dimensional manifold of scalar differential operators (or Lax operators) with $T$-periodic coefficients of the form

$$
L=\frac{d^{n}}{d \theta^{n}}+u_{n-2} \frac{d^{n-2}}{d \theta^{n-2}}+\cdots+u_{1} \frac{d}{d \theta}+u_{0},
$$

and let $\xi_{L}=\left(\xi_{1}, \ldots, \xi_{n}\right)$ be a solution curve associated to $L$ the Wronskian of whose components equals one. Due to the periodicity of the coefficients of $L$, there exists a matrix $M_{L} \in \operatorname{SL}(n, \mathbf{R})$, called the monodromy of $L$, such that

$$
\xi_{L}(\theta+T)=M_{L} \xi_{L}(\theta), \text { for all } \theta \in \mathbf{R} .
$$

( $M_{L}$ is defined by the Floquet matrix of the differential equation.) This same property holds for its (non-degenerate) projection on the $n-1$ sphere $S^{n-1}\left(\hat{\xi}_{L}=\left(\xi_{L} /\left|\xi_{L}\right|\right)\right.$, where $|\cdot|$ represents the 
norm on $\mathbf{R}^{n}$ ), and is also shared by the projective coordinates of this projection, whenever we consider the actions of $\operatorname{SL}(n, \mathbf{R})$ on the sphere and on projective space, respectively. Observe that the monodromy is not completely determined by the operator $L$, but by its solution curves. Namely, if one chooses a different solution curve, its monodromy won't be equal to $M_{L}$ in general, but it will be the conjugate of $M_{L}$ by an element of $\operatorname{GL}(n, \mathbf{R})$. That is, $L$ only determines the conjugation class of the monodromy. Of course, this problem does not exist once the solution curve has been fixed.

Conversely, let $\phi: \mathbf{R} \rightarrow \mathbf{R} \mathbf{P}^{n-1}$ be a curve on $\mathbf{R} \mathbf{P}^{n-1}$. Assume that the curve $\phi$ is nondegenerate and right-hand oriented, that is the Wronskian determinant of the components of its derivative $\phi^{\prime}$ is positive. (This is equivalent to the Wronskian of the components of $(1, \phi)$ being positive; for example, the curve would be convex and right-hand oriented in the case $n=3$.) Assume also that $\phi$ satisfies the following monodromy property:

$$
\phi(\theta+T)=(M \phi)(\theta), \quad \text { for all } \theta \in \mathbf{R},
$$

for a given $M \in \operatorname{SL}(n, \mathbf{R})$. Here $M \phi$ represents the usual action of $\operatorname{SL}(n, \mathbf{R})$ on $\mathbf{R} \mathbf{P}^{n-1}$, induced by the action of $\operatorname{SL}(n, \mathbf{R})$ on $\mathbf{R}^{n}$. One can associate to $\phi$ a differential operator of the form (2.1) in the following manner: We lift $\phi$ to a curve on $\mathbf{R}^{n}$, say to $f(\theta)(1, \phi)$. We choose the factor $f$ so that the Wronskian of the components of the new curve equals 1 . There is a unique choice of $f$ with such a property (up to perhaps a sign), namely

$$
f=W\left(1, \phi_{1}, \ldots, \phi_{n-1}\right)^{-1 / n}=W\left(\phi_{1}^{\prime}, \ldots, \phi_{n-1}^{\prime}\right)^{-1 / n},
$$

where $\phi=\left(\phi_{1}, \ldots, \phi_{n-1}\right)$ and $W$ represents the Wronskian determinant.

It is not very hard to see that the coordinate functions of the lifted curve are solutions of a unique differential operator of the form (2.1). Such an operator defines an equation for an unknown $y$ of the form

$$
\left|\begin{array}{cccc}
y & f \phi_{0} & \ldots & f \phi_{n-1} \\
y^{\prime} & \left(f \phi_{0}\right)^{\prime} & \ldots & \left(f \phi_{n-1}\right)^{\prime} \\
\vdots & \vdots & \ddots & \vdots \\
y^{(n)} & \left(f \phi_{0}\right)^{(n)} & \ldots & \left(f \phi_{n-1}\right)^{(n)}
\end{array}\right|=0 ; \quad \phi_{0}=1, \quad '=\frac{d}{d \theta} .
$$

Equation (2.3) can be written in the usual manner as a system of first order differential equations $d X / d \theta=N X$, where

$$
N=\left(\begin{array}{ccccc}
0 & 1 & 0 & \ldots & 0 \\
0 & 0 & 1 & \ldots & 0 \\
\vdots & \vdots & \ddots & \ddots & \vdots \\
0 & 0 & \ldots & 0 & 1 \\
-u_{0} & -u_{1} & \ldots & -u_{n-2} & 0
\end{array}\right)
$$

and $X$ is a fundamental matrix solution associated to the differential equation (2.3). From this formulation and the monodromy condition it is trivial to see that $N=(d X / d \theta) X^{-1}$ is a periodic matrix and so are the coefficients of the operator defining (2.3).

A short comment is due at this point: if $M$ is the monodromy matrix associated to $\phi$, for even $n$ the monodromy matrix associated to $L$ could be either $M$ or $-M$, depending on whether the first component of $M(1, \phi)$ is positive or negative. Hence, it would be more correct to talk about the action of $\operatorname{PSL}(n, \mathbf{R})$, the space obtained from $\operatorname{SL}(n, \mathbf{R})$ by identifying $M$ and $-M$. Since this choice makes no difference in what follows, we will keep $\operatorname{SL}(n, \mathbf{R})$ for the sake of simplicity. 


\section{THE EVOLUTION EQUATIONS ON $\mathscr{b}_{n}$}

The Adler-Gel'fand-Dikii bracket. We start by describing one of the Hamiltonian evolutions on the manifold $\mathscr{b}_{n}$, the well known Adler-Gel'fand-Dikii bracket, or second KdV Hamiltonian structure.

Given a linear functional $\mathscr{H}$ on $\mathscr{A}_{n}$, one can associate to it a pseudo-differential operator

$$
H=\sum_{i=1}^{n} h_{i} \partial^{-1}, \quad \partial=\frac{d}{d \theta},
$$

such that

$$
\mathscr{H}(L)=\int_{S^{1}} \operatorname{res}(H L) d \theta,
$$

where res selects the coefficient of $\partial^{-1}$ and is called the residue of the pseudo-differential operator (see Ref. 1 or 2 ). To any $\mathscr{H}$ we can associate a (Hamiltonian) vector field $V_{H}$ defined as

$$
V_{H}(L)=(L H)_{+} L-L(H L)_{+},
$$

where by $(\cdot)_{+}$we denote the non-negative (or differential) part of the operator. The map $H \rightarrow V_{H}$ is a structure map defining a Poisson bracket on the manifold $\mathscr{A}_{n}$. If $\hat{l}$ is the matrix of differential operators defining the structure map, the Poisson bracket is defined as

$$
\{\mathscr{H}, \mathscr{F}\}(L)=\int_{S^{1}} \operatorname{res}(\hat{l}(H) F) d \theta
$$

cf. Refs. 1, 2 or 10. The original definition of the bracket was given by Adler, ${ }^{1}$ in an attempt to make generalized KdV equations bi-Hamiltonian systems. Gel'fand and Dikii proved Jacobi's identity in Ref. 2. In the case $n=2$, this bracket coincides with the Lie-Poisson structure on the dual of the Virasoro algebra. Two other equivalent definitions of the original bracket were found in Refs. 3 and 4. The original definition is rather complicated, so we will explain and use the one in Ref. 3.

The Kupershmidt-Wilson bracket. In a very interesting paper, ${ }^{3}$ Kupershmidt and Wilson gave an equivalent but rather simpler definition of the bracket (3.1). Consider $L$ to be an operator of the form (2.1). Assume that the operator $L$ factors into a product of first-order factors

$$
L=\left(\partial+y_{n-1}\right)\left(\partial+y_{n-2}\right) \cdots\left(\partial+y_{1}\right)\left(\partial+y_{0}\right) \text {, }
$$

where

$$
y_{k}=\omega^{k} v_{1}+\omega^{2 k} v_{2}+\cdots+\omega^{(n-1) k} v_{n-1}, \quad 0 \leqslant k \leqslant n-2 ; \quad \omega=e^{2 \pi i / n},
$$

and $y_{n-1}=-\sum_{i=0}^{n-2} y_{i}$. The variables $v_{i}, 1 \leqslant i \leqslant n-1$, are what Kupershmidt and Wilson called "modified" variables. Even though the factorization is not unique (and so some reduction had to be involved in the proof of Ref. 3), one can find a unique factorization once a solution curve has been fixed, as we will see later.

Assume that the coefficients $u_{i}, 0 \leqslant i \leqslant n-2$, of $L$ evolve following a Hamiltonian evolution with respect to the second $\mathrm{KdV}$ Hamiltonian structure. The result in Ref. 3 then states that the corresponding "'modified"' coordinates $v_{i}$ evolve following a Hamiltonian evolution with respect to a Poisson bracket defined by the structure map 


$$
l=-\frac{1}{n} \partial J,
$$

where

$$
J=\left(\begin{array}{cccc}
0 & \ldots & 0 & 1 \\
0 & \ldots & 1 & 0 \\
\vdots & . \cdot & . \cdot & \vdots \\
1 & 0 & \ldots & 0
\end{array}\right)
$$

That is,

$$
\frac{D u}{D v} l\left(\frac{D u}{D v}\right)^{*}=\hat{l},
$$

where $D u / D v=\left(D u_{i} / D v_{j}\right)$,

$$
\frac{D u_{i}}{D v_{j}}=\sum_{k=0}^{n-1} \frac{\partial u_{i}}{\partial v_{j}^{(k)}} \partial^{k}
$$

being the Fréchet derivative of $u_{i}$ with respect to $v_{j}$. Also, by * we denote the adjoint matrix operator, the transposed of the matrix whose entries are the adjoint operators of the entries of the original matrix. Thus, the original Adler-Gel'fand-Dikii bracket arises from a very simple bracket defined on the space of "modified" variables $v$.

Many facts are known about this Hamiltonian structure. Since it is Poisson (degenerate), the manifold $\mathscr{C}_{n}$ foliates into symplectic leaves, maximal submanifolds where the Hamiltonian flow always lies. These leaves are classified locally by the conjugation class of the monodromy matrix associated to the operators lying on the leaf. In other words, if two operators are close and have conjugate monodromies, there is a Hamiltonian path joining them. There exists another discrete invariant that classifies the leaves globally, cf. Ref. 9, based on topological properties of the projection of the solution curves on the sphere $S^{n}$.

\section{INVARIANT EVOLUTION EQUATIONS ON $\mathscr{C}_{n}$}

The duality between $\mathscr{C}_{n}$ and $\mathscr{C}_{n}$ described in the previous sections makes it natural to study evolution equations on the space $\mathscr{C}_{n}$ whose associated flow leaves the Adler-Gel'fand-Dikii symplectic leaves invariant. In other words, we are interested in partial differential equations of the form

$$
\phi_{t}=F\left(\theta, \phi, \phi_{\theta}, \phi_{\theta \theta}, \ldots\right), \quad \phi: \mathbf{R}^{2} \rightarrow \mathbf{R} \mathbf{P}^{n-1},
$$

for a function $\phi(\theta, t)$, with the property that, if the initial condition has a monodromy property (2.2), then every solution $\phi(\cdot, t)$ of $(4.1)$ has also a monodromy property, and the conjugation class of the monodromy matrix is independent of $t$. The simplest evolution equations having this property are those of the form (4.1) with $F$ independent of $\theta$ which are also invariant under the standard projective action of $\operatorname{SL}(n, \mathbf{R})$ on the dependent variables $\phi=\left(\phi_{1}, \ldots, \phi_{n-1}\right)$. In other words, we are dealing with equations of the form

$$
\phi_{t}=F\left(\phi, \phi_{\theta}, \phi_{\theta \theta}, \ldots\right), \quad \phi: \mathbf{R}^{2} \rightarrow \mathbf{R P}^{n-1},
$$

such that whenever $\phi(\theta, t)$ is a solution of (4.2) so is $(M \phi)(\theta, t)$, for all $M \in \operatorname{SL}(n, \mathbf{R})$. To see that the monodromy class of the solutions $\phi(\cdot, t)$ of an equation (4.2) invariant under the action of 
$\operatorname{SL}(n, \mathbf{R})$ is indeed preserved under the evolution, note that (4.2) is also invariant under translations of the independent variable $\theta$. Hence, if the initial condition $\phi(\cdot, 0)$ of $(4.2)$ has a matrix $M \in \operatorname{SL}(n, \mathbf{R})$ as monodromy, and we consider a different curve in the flow $\phi(\cdot, t)$, we have that $\phi(\theta-T, t)$ is also a solution. If (4.2) is $\operatorname{SL}(n, \mathbf{R})$-invariant, $M \phi(\theta-T, t)$ will also be a solution of (4.2). Applying uniqueness of solutions of (4.2) (whenever possible), $M \phi(\theta-T, t)=\phi(\theta, t)$, so that $\phi(\cdot, t)$ has the same monodromy as $\phi(\cdot, 0)$. If there is no uniqueness of solutions, both Hamiltonian and invariant evolutions are obviously much more complicated; we won't deal with those cases in this paper.

Remark: note that the evolution associated to an $\operatorname{SL}(n, \mathbf{R})$-invariant equation (4.2) preserves exactly the monodromy (not just the monodromy class) of its solutions.

In this paper we conjecture that the Adler-Gel'fand'-Dikii evolution on $\mathscr{b}_{n}$ and the $\operatorname{SL}(n, \mathbf{R})$-invariant evolution (4.2) on $\mathscr{C}_{n}$ are identical under the identification described in the Introduction, provided that the coefficients of the Hamiltonian $H$ (the pseudo-differential operator describing the differential of the functional $\mathscr{H}$ ) are equal to a vector differential invariant of the projective action. We will find the most general $\operatorname{SL}(n, \mathbf{R})$-invariant evolution of the form (4.2), showing then how the conjecture can be proved for a number of values of $n$ and where the main problem lies in the proof of the general case.

The most general evolution equation of the form (4.2) invariant under the projective action

$$
\phi(\theta, t) \mapsto(M \phi)(\theta, t)
$$

of $\operatorname{SL}(n, \mathbf{R})$ can be found using the general infinitesimal techniques described in Refs. 10, 6. First of all, the infinitesimal generators of the projective $\operatorname{SL}(n, \mathbf{R})$ action are easily found to be the following vector fields on $\mathbf{R} \times \mathbf{R} \times \mathbf{R} \mathbf{P}^{n-1}$ :

$$
\mathbf{v}_{i}=\frac{\partial}{\partial \phi_{i}}, \quad \mathbf{v}_{i j}=\phi_{i} \frac{\partial}{\partial \phi_{j}}, \quad \mathbf{w}_{i}=\phi_{i} \sum_{j=1}^{n} \phi_{j} \frac{\partial}{\partial \phi_{j}} ; \quad 1 \leqslant i, j \leqslant n-1 .
$$

The vector fields (4.3) are a basis of a realization of the Lie algebra $\mathfrak{s l}(n, \mathbf{R})$. Note that all these vector fields are independent of the variables $(\theta, t)$, and they are also "vertical," i.e., their $\theta$ and $t$ components vanish.

If $\mathbf{v}=\sum_{i=1}^{n-1} \eta_{i}(\theta, t, \phi) \partial / \partial \phi_{i}$ is a vertical vector field, its prolongation is the vector field $\mathrm{pr} \mathbf{v}$ defined by

$$
\operatorname{pr} \mathbf{v}=\mathbf{v}+\sum_{\substack{j \geqslant 1 \\ k \geqslant 0}} \sum_{i=1}^{n-1}\left(D_{t}^{k} D^{j} \eta_{i}\right) \frac{\partial}{\partial\left(\partial_{t}^{k} \phi_{i}^{(j)}\right)},
$$

where $\phi_{i}^{(j)}=\partial^{j} \phi_{i}, D$ is the total derivative operator with respect to $\theta$

$$
D=\partial+\sum_{j \geqslant 0} \sum_{i=1}^{n-1} \phi_{i}^{(j+1)} \frac{\partial}{\partial \phi_{i}^{(j)}},
$$

and

$$
D_{t}=\partial_{t}+\sum_{j \geqslant 0} \sum_{i=1}^{n-1}\left(\partial_{t} \phi_{i}^{(j)}\right) \frac{\partial}{\partial \phi_{i}^{(j)}}
$$

is the total derivative operator with respect to $t$. In general, the vector field $\operatorname{pr} \mathbf{v}$ is defined on the infinite-dimensional jet space $J^{\infty}\left(\mathbf{R} \times \mathbf{R}, \mathbf{R} \mathbf{P}^{n-1}\right)$ with local coordinates $\theta, t, \partial_{t}^{k} \phi_{i}^{(j)} \quad(1 \leqslant i \leqslant n$ $-1 ; k, j \geqslant 0$ ). However, when pr $\mathbf{v}$ is applied to a function (like $F$ ) independent of the coordinates $\partial_{t}^{k} \phi_{i}^{(j)}(k \geqslant 1)$ involving explicitly $t$-derivatives, (4.4) reduces to the vector field 


$$
\operatorname{pr} \mathbf{v}=\mathbf{v}+\sum_{j \geqslant 1} \sum_{i=1}^{n-1}\left(D^{j} \eta_{i}\right) \frac{\partial}{\partial \phi_{i}^{(j)}},
$$

defined on the infinite-dimensional jet space $J^{\infty} \equiv J^{\infty}\left(\mathbf{R}, \mathbf{R P}^{n-1}\right)$ with local coordinates $\theta, \phi_{i}^{(j)}$ $(1 \leqslant i \leqslant n-1, j \geqslant 0)$. Following Ref. 10, we can express the necessary and sufficient condition for (4.2) to be invariant under the action of $\operatorname{SL}(n, \mathbf{R})$ "infinitesimally" as follows:

$$
\operatorname{pr} \mathbf{v}(F)=\left.D_{t} \eta\right|_{\phi_{t}=F}, \quad \text { for all } \mathbf{v}=\sum_{i=1}^{n-1} \eta_{i}(\phi) \frac{\partial}{\partial \phi_{i}} \in \mathfrak{s l}(n, \mathbf{R}) .
$$

Note that, although both $\operatorname{pr} \mathbf{v}, D$ and $D_{t}$ are formally defined on infinite-dimensional jet spaces, in practice they will always act on functions depending on a finite number of the local coordinates. Finally, using the fact that $\eta$ is a function of $\phi$ only and (4.5), equation (4.7) can be further simplified as follows:

$$
\operatorname{pr} \mathbf{v}(F)=\frac{\partial \eta}{\partial \phi} F
$$

where $\partial \eta / \partial \phi$ is the $(n-1) \times(n-1)$ matrix with $(i, j)$ entry $\partial \eta_{i} / \partial \phi_{j}$. In other words, $F$ is a relative vector differential invariant of the Lie algebra $\mathfrak{s l}(n, \mathbf{R})$ given by (4.3), whose associated weight is the matrix $\partial \eta / \partial \phi$. Using standard techniques (cf. Ref. 6), we can give the following characterization of the general solution of (4.8):

4.1 Theorem: The most general solution $F$ of equation (4.8) is of the form

$$
F=\mu \mathscr{T},
$$

where the $(n-1) \times(n-1)$ matrix $\mu=\left(\mu^{1} \mu^{2} \cdots \mu^{n-1}\right)$ is any matrix with non-vanishing determinant and whose columns $\mu^{i}$ are particular solutions of (4.8), and $\mathscr{T}=\left(\mathscr{T}_{k}\right)_{k=1}^{n-1}$ is an arbitrary absolute (vector) differential invariant of the algebra (4.3), i.e., a solution of

$$
\operatorname{pr} \mathbf{v}\left(\mathscr{T}_{i}\right)=0, \quad \text { for all } \mathbf{v} \in \mathfrak{s l}(n, \mathbf{R}), \quad i=1, \ldots, n-1
$$

The problem of calculating the most general absolute differential invariant $\mathscr{T}$ of a given Lie algebra of vector fields is a classical one ${ }^{11-13}$ whose solution in a modern formulation can be found in Ref. 6 . The general result asserts that their exist $n$ functionally independent differential fundamental invariants $J_{0}, J_{1}, \ldots, J_{n-1}$, such that any differential invariant is a function of the $J_{i}$ 's and their "covariant derivatives" $\mathscr{D}^{k} J_{i}$, where $\mathscr{D}=\left(D J_{0}\right)^{-1} D$. Since in our case the generators (4.3) are independent of $\theta$, we can take $J_{0}=\theta$, so that the operator $\mathscr{D}$ reduces to $D$ in this case. Therefore, we can state the following Theorem:

4.2 Theorem: The most general ( $\theta$-independent) absolute differential invariant of the $\mathfrak{s l}(n, \mathbf{R})$ Lie algebra (4.3) is a function of $n-1$ fundamental differential invariants $J_{i}\left(\phi, \ldots, \phi^{(m)}\right)$ and their total derivatives with respect to $\theta$.

For $n=2$, it is straightforward to compute the fundamental $\mathfrak{s l}(2, \mathbf{R})$ invariant $J_{1}$. The result is the classical Schwartzian derivative $S(\phi)$ of $\phi$ :

$$
J_{1}=\frac{\phi^{\prime \prime \prime}}{\phi^{\prime}}-\frac{3}{2} \frac{\phi^{\prime \prime 2}}{\phi^{\prime 2}} .
$$

In this case, the matrix $\partial \eta / \partial \phi$ is just a function, which makes a simple matter to find a particular vector differential invariant of weight $\partial \eta / \partial \phi$. The simplest such invariant is $\phi^{\prime} \equiv \phi_{\theta}$; therefore, Theorem 4.2 implies the following: 
4.3 Theorem: For $n=2$, the most general evolution equation (4.2) invariant under the projective action of $\operatorname{SL}(n, \mathbf{R})$ is

$$
\phi_{t}=\phi_{\theta} \mathscr{T}\left(S, D S, \ldots, D^{l} S\right),
$$

where $S$ is the Schwartzian derivative of $\phi(\cdot, t)$, and $\mathscr{T}$ is an arbitrary (smooth) function.

Even for the case $n=3$, it is not an easy matter to find the $n-1$ fundamental differential invariants of (4.3) and a particular matrix relative differential invariant of weight $\partial \eta / \partial \phi$ from scratch. Fortunately, however, the differential invariants of the projective action of $\operatorname{SL}(n, \mathbf{R})$ have been the object of considerable study in classical projective differential geometry. ${ }^{14}$ From this viewpoint, the differential invariants of a projective curve describe the properties of the curve invariant under the group of motions of projective space, or in other words the properties of the curve independent of the particular system of projective coordinates used to represent it. An intrinsic description of a projective curve must therefore be done in terms of its $\mathfrak{s l}(n, \mathbf{R})$ differential invariants. It is not hard to see (as we will explain in the following section) that the coefficients of the operator $L$ defined by a projective curve $\phi$ as in (2.3) are a set of functionally independent differential invariants. Obviously, they determine the curve up to a projective transformation; this was already known to Wilczynski ${ }^{14}$ and it is a generalization of the well known result in Euclidean geometry that the curvatures of a curve in Euclidean space, expressed as functions of the Euclidean-invariant arclength, uniquely characterize the curve up to an Euclidean motion. We shall explain in the following sections how this equivalence between fundamental differential invariants and coefficients of the operator $L$ is the key to the duality of evolutions.

\section{THE EXPLICIT FORMULA FOR THE SL $(n, R)$ INVARIANT EVOLUTION}

In this section we will describe a complete set of independent differential invariants for the projective action of $\operatorname{SL}(n, \mathbf{R})$, and we will give the explicit expression of the relative invariant (4.8) with the required weight, for arbitrary $n$. The complete set of differential invariants was already found by Ref. 14 and is precisely given by the coefficients of the operator $L$ determined by the curve $\phi$, as mentioned in the Introduction.

5.1 Theorem: Let $\phi$ be a non-degenerate and right-hand oriented curve on $\mathbf{R P}^{n-1}$, and let

$$
L=\frac{d^{n}}{d \theta^{n}}+u_{n-2} \frac{d^{n-2}}{d \theta^{n-2}}+\cdots+u_{1} \frac{d}{d \theta}+u_{0}
$$

be the differential operator determined by $\phi$ through the relation (2.3). Then the coefficients $u_{i}$, $0 \leqslant i \leqslant n-2$, form a complete set of functionally independent differential invariants for the $\mathrm{SL}(n, \mathbf{R})$ action on $\mathbf{R P}^{n-1}$.

Proof: Using the form of equation (2.3) one can easily see that the coefficient of $d^{k} / d \theta^{k}$ is given by $u_{k}=-\Delta_{k}$, where $\Delta_{k}$ is the determinant obtained from the Wronskian determinant $W\left(f, f \phi_{1}, \ldots, f \phi_{n-1}\right)=1$ when we substitute the $(k+1)$ th row by the $n$th derivative row $\left(f^{(n)},\left(f \phi_{1}\right)^{(n)}, \ldots,\left(f \phi_{n-1}\right)^{(n)}\right)$. Thus, the coefficients of $L$ are functions of the components of the curve $\phi$ and their derivatives. From this it follows that the coefficients of the operator $L$ are functionally independent functions. Indeed, if there were a functional relation among these functions one could choose an operator whose coefficients did not satisfy this relation. The projectivization $\phi$ of the solution curve of such an operator would then have coefficients $u_{k}(\phi), k$ $=0, \ldots, n-2$, not satisfying the functional relation, and we would get a contradiction.

The coefficients $u_{i}$ are easily shown to be invariants. Indeed, let $M \in \operatorname{SL}(n, \mathbf{R})$ and let $M \phi$ be the image of the curve $\phi$ under the projective action of $M$. If we lift $\phi$ to a solution curve of $L$, say $(f, f \phi)$, and we also lift the curve $M \phi$, we see that the latter is simply $M \cdot(f, f \phi)$ (the dot denoting matrix multiplication). Since $M \cdot(f, f \phi)$ represents a non-degenerate linear combination 
of the solution curve $(f, f \phi)$, both lifted curves are solutions of the same operator and hence $u_{k}(\phi)=u_{k}(M \phi)$ for all $k$.

Q.E.D.

Next, we will find the explicit expression for $n$ independent relative vector invariants, solutions of (4.8) for all vector fields $\mathbf{v}=\sum_{i=1}^{n-1} \eta_{i}(\phi) \partial / \partial \phi_{i} \in \mathfrak{s l}(n, \mathbf{R})$. That is, we want to find a matrix

$$
\mu=\left(\mu^{1} \mu^{2} \ldots \mu^{n-1}\right)
$$

each of whose columns $\mu^{i}$ is a solution of equation (4.8), and such that the determinant of $\mu$ does not vanish.

Before going into the details of how one finds this matrix, we need several preliminary definitions and results:

5.2 Definition: For $i_{1}, \ldots, i_{k} \geqslant 0$ and $1 \leqslant k \leqslant n-1$, let us denote

$$
w_{i_{1} i_{2} \ldots i_{k}}=\left|\begin{array}{cccc}
\phi_{1}^{\left(i_{1}\right)} & \phi_{2}^{\left(i_{1}\right)} & \ldots & \phi_{k}^{\left(i_{1}\right)} \\
\phi_{1}^{\left(i_{2}\right)} & \phi_{2}^{\left(i_{2}\right)} & \ldots & \phi_{k}^{\left(i_{2}\right)} \\
\vdots & \vdots & \ddots & \vdots \\
\phi_{1}^{\left(i_{k}\right)} & \phi_{2}^{\left(i_{k}\right)} & \ldots & \phi_{k}^{\left(i_{k}\right)}
\end{array}\right|
$$

and

$$
W_{k}=w_{12 \ldots k} .
$$

We define the homogeneous variables $q_{i_{1} i_{2} \ldots i_{k}}$ by

$$
q_{i_{1} i_{2} \ldots i_{k}}=\frac{w_{i_{1} i_{2} \ldots i_{k}}}{W_{k}} .
$$

Finally, for $k=1,2, \ldots, n$ the variables $q_{n}^{k}$ are defined as follows:

$$
q_{n}^{k}=q_{12 \ldots \hat{k} \ldots n},
$$

where the notation $\hat{k}$ means that the index $k$ is to be omitted.

The following statements follow easily from elementary properties of determinants:

5.3 Lemma:

(i) For any $k, i_{1}, \ldots, i_{r} \geqslant 0$ and $1 \leqslant s<r \leqslant n-1$ we have the following identities:

$$
\begin{gathered}
q_{k} q_{i_{1} i_{2} \ldots i_{r}}=q_{i_{1}} q_{k i_{2} \ldots i_{r}}+q_{i_{2}} q_{i_{1} k i_{3} \ldots i_{r}}+\cdots+q_{i_{r}} q_{i_{1} \ldots i_{r-1} k}, \\
q_{i_{1} i_{2} \ldots i_{s} k} q_{i_{1} i_{2} \ldots i_{r}}=q_{i_{1} \ldots i_{s} i_{s+1}} q_{i_{1} \ldots i_{s} k i_{s+2} \ldots i_{r}}+q_{i_{1} \ldots i_{s} i_{s+2}} q_{i_{1} \ldots i_{s+1}} k_{i_{s+3} \ldots i_{r}}+\cdots+q_{i_{1} \ldots i_{s} i_{r}} q_{i_{1} \ldots i_{r-1} k} .
\end{gathered}
$$

(ii) If we define $q_{m}^{0}=0$ for all $m \geqslant 2$, then the following identity holds:

$$
q_{n}^{k}=q_{n-1}^{k} q_{n}^{n-1}-q_{n-1}^{k} q_{n-1}^{n-2}-\left(q_{n-1}^{k}\right)^{\prime}+q_{n-1}^{k-1}, \quad 1 \leqslant k<n .
$$

Note that $q_{n}^{n}=1$ by definition. The affine algebra is the subalgebra of the $\mathfrak{s l}(n, \mathbf{R})$ algebra (4.3) generated by the vector fields $\mathbf{v}_{r}$ and $\mathbf{v}_{r s}, 1 \leqslant r, s \leqslant n-1$. The corresponding group of transformations is the affine group, i.e., the semidirect product of the translation group with the general linear group in the variables $\left(\phi_{1}, \ldots, \phi_{n-1}\right)$.

5.4 Lemma: If a $\theta$-independent function $\psi: J^{\infty} \equiv J^{\infty}\left(\mathbf{R}, \mathbf{R} \mathbf{P}^{n-1}\right) \rightarrow \mathbf{R}$ is invariant under the action of the affine algebra, then $\psi$ necessarily depends only on the affine coordinates $q_{n}^{r}, r$ $=1, \ldots, n-1$, and their derivatives. 
Proof: Consider the prolonged action of the affine algebra on the $k$ th jet space $J^{k}$ $\equiv J^{k}\left(\mathbf{R}, \mathbf{R} \mathbf{P}^{n-1}\right)$, whose infinitesimal generators are the $k$ th prolongations (i.e., the truncations of the prolongations (4.6) at differential order $k$ )

$$
\operatorname{pr}^{(k)} \mathbf{v}_{r}=\mathbf{v}_{r}, \quad \operatorname{pr}^{(k)} \mathbf{v}_{r s}=\sum_{j=0}^{k} \phi_{r}^{(j)} \frac{\partial}{\partial \phi_{s}^{(j)}}, \quad 1 \leqslant r, s<n-1 .
$$

For $k \leqslant n-1$, at a generic point of $J^{k}$ the $n(n-1)$ vector fields (5.3) span the $(k+1)(n-1)$-dimensional subspace of the tangent space of $J^{k}$ whose elements are the "vertical" vector fields (whose component along $\partial / \partial \theta$ vanishes). By Frobenius theorem, this implies that there are no affine differential invariants of differential order between 1 and $n-1$, and the only zeroth order invariant is clearly (a function of) the coordinate $\theta$. It is also immediate to check that for $k \geqslant n-1$ the vector fields (5.3) are linearly independent at a generic point. Hence the maximal dimension of the span of these vector fields stabilizes for $k=n-1$. Olver's general results, cf. Ref. 6 , imply that the affine algebra has $n-1$ fundamental invariants of order $n$, and that an arbitrary differential invariant can be expressed as a function of $\theta$, the fundamental invariants, and their derivatives with respect to the zeroth order invariant $\theta$. Since the $n-1$ functions $q_{n}^{r}, 1 \leqslant r \leqslant n$ -1 , all have differential order $n$, and are clearly functionally independent and invariant under general affine transformations of the variables $\left(\phi_{1}, \ldots, \phi_{n-1}\right)$ by their definition, they can be taken as the $n-1$ fundamental invariants.

Q.E.D.

5.5 Lemma: The variables $q_{r}^{s}(r>s \geqslant 1)$ can be written in terms of the functionally independent functions $q_{k}^{k-1}(k \geqslant 2)$ and their derivatives. We will call the latter functions basic homogeneous variables.

Proof: We will prove the lemma by induction on $r-s$. For $r-s=1$, the lemma holds trivially. Assume now that the functions $q_{r^{\prime}}^{s^{\prime}}$ with $r^{\prime}-s^{\prime}<m$ can be expressed in terms of the functions $q_{k}^{k-1}$ and their derivatives. Let $q_{r}^{s}$ be such that $r-s=m$. From (ii) of Lemma 5.3 we have that

$$
q_{r}^{s}=q_{r-1}^{s} q_{r}^{r-1}-q_{r-1}^{s} q_{r-1}^{r-2}-\left(q_{r-1}^{s}\right)^{\prime}+q_{r-1}^{s-1},
$$

so that by the induction hypothesis $q_{r}^{s}$ can be written in terms of the functions $q_{k}^{k-1}$ and their derivatives if, and only if, the same is true for $q_{r-1}^{s-1}$. Repeating this argument $s-2$ times, we see that $q_{r}^{s}$ will be a function of the $q_{k}^{k-1}$ and their derivatives, if and only if this is the case for $q_{m+1}^{1}$, with $m=r-s>0$. Again from (ii) in Lemma 5.3, we have that

$$
q_{m+1}^{1}=q_{m}^{1} q_{m+1}^{m}-q_{m}^{1} q_{m}^{m-1}-\left(q_{m}^{1}\right)^{\prime}
$$

which, by the induction hypothesis, proves the lemma.

Q.E.D.

We are now going to make an ansatz for the matrix $\mu$. Namely, we will look among matrices $\mu$ of the form

$$
\mu=\Phi(\operatorname{Id}+A)
$$

where

$$
\Phi=\left(\begin{array}{cccc}
\phi_{1}^{\prime} & \phi_{1}^{\prime \prime} & \cdots & \phi_{1}^{(n-1)} \\
\phi_{2}^{\prime} & \phi_{2}^{\prime \prime} & \cdots & \phi_{2}^{(n-1)} \\
\vdots & \vdots & \ddots & \vdots \\
\phi_{n-1}^{\prime} & \phi_{n-1}^{\prime \prime} & \cdots & \phi_{n-1}^{(n-1)}
\end{array}\right)
$$


Id is the identity matrix, and $A$ is a strictly upper triangular matrix to be determined. Obviously, a matrix $\mu$ of this form will have a nonvanishing determinant.

5.6 Theorem: An invertible matrix $\mu$ of relative invariants with weight $\partial \eta / \partial \phi$ is given by a matrix of the form (5.4)-(5.5), with $A=\left(a_{i}^{j}\right)$ defined by

$$
a_{i}^{j}=\left\{\begin{array}{l}
\frac{(-1)^{j-i}\left(\begin{array}{l}
i \\
j
\end{array}\right)}{\left(\begin{array}{l}
n \\
j-i
\end{array}\right)} q_{n}^{n-j+i}, \quad i<j \\
0, \quad i \geqslant j
\end{array} .\right.
$$

Proof: We only need to show that each one of the columns of $\mu$ is a particular solution of equation (4.8). Assume that $\mu=\left(\mu^{1} \ldots \mu^{n-1}\right)$ is of the form (5.4)-(5.5), so that $\mu^{i}=\left(\mu_{j}^{i}\right)_{j=1}^{n-1}$ is a column given by $\mu_{j}^{i}=\phi_{j}^{(i)}+\sum_{k=1}^{i-1} a_{k}^{i} \phi_{j}^{(k)}$. Assume also that $\mathbf{v}=\sum_{i=1}^{n-1} \eta_{i}(\phi) \partial / \partial \phi_{i} \in \mathfrak{s l}(n, \mathbf{R})$. We can then write equation (4.8) as

$$
\operatorname{pr} \mathbf{v}\left(\mu_{j}^{i}\right)=\sum_{k=1}^{n-1} \frac{\partial \eta_{j}}{\partial \phi_{k}} \mu_{k}^{i}
$$

Obviously, it suffices that (5.7) hold for all the basic vector fields (4.3). We will therefore consider the following three cases:

(a) If $\mathbf{v}=\mathbf{v}_{r}=\partial / \partial \phi_{r}$, then pr $\mathbf{v}=\mathbf{v}$ and (5.7) trivially holds, since both sides of the equality vanish.

(b) If $\mathbf{v}=\mathbf{v}_{r s}=\phi_{r}\left(\partial / \partial \phi_{s}\right)$, then its prolongation is given by $\operatorname{pr} \mathbf{v}_{r s}=\Sigma_{k \geqslant 0} \phi_{r}^{(k)}\left(\partial / \partial \phi_{s}^{(k)}\right)$. Substituting in (5.7), we obtain the equivalent equation

$$
\sum_{k=1}^{i-1} \phi_{j}^{(k)} \operatorname{pr} \mathbf{v}_{r s}\left(a_{k}^{i}\right)=0, \quad 1 \leqslant i, j, r, s \leqslant n-1
$$

In matrix notation the latter equation becomes

$$
\Phi \operatorname{pr} \mathbf{v}_{r s}(A)=0, \quad r, s=1,2, \ldots, n-1,
$$

and since $\Phi$ is invertible for all projective curves under consideration this is equivalent to

$$
\operatorname{pr} \mathbf{v}_{r s}(A)=0, \quad r, s=1,2, \ldots, n-1 .
$$

(By $\operatorname{pr} \mathbf{v}_{r s}(A)$ we mean the matrix obtained when we apply the vector field pr $\mathbf{v}_{\tau s}$ to each of the entries of the matrix $A$.) Since the matrix $A$ in (5.6) depends only on the affine invariant coordinates $q_{n}^{r}, 1 \leqslant r \leqslant n-1$, by Lemma 5.4 we deduce that (5.8) holds for this matrix.

(c) If $\mathbf{v}=\mathbf{w}_{r}=\phi_{r} \sum_{k=1}^{n-1} \phi_{k}\left(\partial / \partial \phi_{k}\right)$, its prolongation is given by the formula

$$
\operatorname{pr} \mathbf{w}_{r}=\sum_{j \geqslant 0} \sum_{k=1}^{n-1}\left(\phi_{r} \phi_{k}\right)^{(j)} \frac{\partial}{\partial \phi_{k}^{(j)}} .
$$

Substituting this formula into (5.7), we easily arrive at the matrix equation

$$
\Phi \operatorname{pr} \mathbf{w}_{r}(A)=\hat{\Phi}_{r}(\mathrm{Id}+A), \quad r=1,2, \ldots, n-1,
$$

where

$$
\left(\hat{\Phi}_{r}\right)_{j}^{i}=\phi_{r} \phi_{j}^{(i)}+\phi_{j} \phi_{r}^{(i)}-\left(\phi_{j} \phi_{r}\right)^{(i)} .
$$


The product $\Phi^{-1} \hat{\Phi}_{r}$ can be easily rewritten in a nice way. In fact, the $(j, i)$ entry of this product is given by

$$
-\sum_{k=1}^{n-1} \phi_{j}^{k} \sum_{l=1}^{i-1}\left(\begin{array}{l}
i \\
l
\end{array}\right) \phi_{k}^{(l)} \phi_{r}^{(i-l)}
$$

where $\phi_{j}^{k}$ is the $(j, k)$ element of $\Phi^{-1}$. Now, since $\sum_{k=1}^{n-1} \phi_{j}^{k} \phi_{k}^{(l)}=\delta_{j}^{l}$, the $(j, i)$ entry of the product $\Phi^{-1} \hat{\Phi}_{r}$ equals zero if $j \geqslant i$ and $-\left(\begin{array}{c}i \\ j\end{array}\right) \phi_{r}^{(i-j)}$ whenever $j<i$. Therefore, the infinitesimal invariance condition in case (c) is given by

$$
\operatorname{pr} \mathbf{w}_{r}(A)=-\Gamma_{r}(\operatorname{Id}+A), \quad r=1,2, \ldots, n-1,
$$

where

$$
\Gamma_{r}=\left(\begin{array}{ccccc}
0 & \left(\begin{array}{l}
2 \\
1
\end{array}\right) \phi_{r}^{\prime} & \left(\begin{array}{l}
3 \\
1
\end{array}\right) \phi_{r}^{\prime \prime} & \ldots & \left(\begin{array}{c}
n-1 \\
1
\end{array}\right) \phi_{r}^{(n-2)} \\
0 & 0 & \left(\begin{array}{l}
3 \\
2
\end{array}\right) \phi_{r}^{\prime} & \ldots & \left(\begin{array}{c}
n-1 \\
2
\end{array}\right) \phi_{r}^{(n-3)} \\
\vdots & \vdots & \ddots & \ddots & \vdots \\
0 & \ddots & 0 & 0 & \left(\begin{array}{c}
n-1 \\
n-2
\end{array}\right) \phi_{r}^{\prime} \\
0 & \ldots & 0 & 0 & 0
\end{array}\right) .
$$

To complete the proof, we only need to check that (5.9) is satisfied when $A$ is given by (5.6). What follows are straightforward calculations.

First of all, one can easily see that

$$
\begin{aligned}
\operatorname{pr} \mathbf{w}_{r}\left(w_{12 \ldots \hat{k} \ldots n}\right)= & \sum_{\substack{j=1 \\
j \neq k}}^{n} \phi_{r} w_{12 \ldots \hat{k} \ldots n}+\sum_{j=1}^{k-1}(-1)^{j-1} \boldsymbol{\phi}_{r}^{(j)} w_{01 \ldots \hat{j} \ldots \hat{k} \ldots n}+\sum_{j=k+1}^{n}(-1)^{j} \boldsymbol{\phi}_{r}^{(j)} w_{01 \ldots \hat{k} \ldots \hat{j} \ldots n} \\
& +\sum_{j=k+1}^{n}\left(\begin{array}{l}
j \\
k
\end{array}\right)(-1)^{j-k+1} \boldsymbol{\phi}_{r}^{(j-k)} w_{12 \ldots \hat{j} \ldots n} .
\end{aligned}
$$

Using formula (5.2) we obtain

$$
\phi_{r} q_{n}^{k}=\sum_{j=1}^{k-1}(-1)^{j-1} \phi_{r}^{(j)} q_{01 \ldots \hat{j} \ldots \hat{k} \ldots n}+\sum_{j=k+1}^{n}(-1)^{j} \phi_{r}^{(j)} q_{01 \ldots \hat{k} \ldots \hat{j} \ldots n},
$$

so that

$$
\operatorname{pr} \mathbf{w}_{r}\left(w_{12 \ldots \hat{k}_{\ldots .}}\right)=n \phi_{r} w_{12 \ldots \hat{k} \ldots n}+\sum_{j=k+1}^{n}\left(\begin{array}{l}
j \\
k
\end{array}\right)(-1)^{j-k+1} \phi_{r}^{(j-k)} w_{12 \ldots \hat{j} \ldots n}
$$

Applying Leibniz's rule we finally obtain

$$
\operatorname{pr} \mathbf{w}_{r}\left(q_{n}^{k}\right)=\sum_{j=k+1}^{n}\left(\begin{array}{l}
j \\
k
\end{array}\right)(-1)^{j-k+1} \phi_{r}^{(j-k)} q_{n}^{j} .
$$


If we substitute in (5.9) the value of $A$ given in the Theorem and use the expression of $\operatorname{pr} \mathbf{w} r\left(q_{n}^{k}\right)$ derived above (5.9) becomes

$$
\begin{gathered}
(-1)^{j-i} \frac{\left(\begin{array}{l}
j \\
i
\end{array}\right)}{\left(\begin{array}{c}
n \\
j-i
\end{array}\right)} \sum_{l=n-j+i+1}^{n}\left(\begin{array}{c}
l \\
n-j+i
\end{array}\right)(-1)^{l-n+j-i+1} \phi_{r}^{(l-n+j-i)} q_{n}^{l} \\
=-\sum_{l=i+1}^{i}\left(\begin{array}{l}
l \\
i
\end{array}\right) \phi_{r}^{(l-i)}(-1)^{j-l} \frac{\left(\begin{array}{l}
j \\
l
\end{array}\right)}{\left(\begin{array}{c}
n \\
j-l
\end{array}\right)} q_{n}^{n-j+l}, \quad 1 \leqslant i<j \leqslant n-1 .
\end{gathered}
$$

This equation will hold provided that

$$
\frac{\left(\begin{array}{l}
j \\
i
\end{array}\right)\left(\begin{array}{l}
n+l-j \\
l-i
\end{array}\right)}{\left(\begin{array}{c}
n \\
j-i
\end{array}\right)}=\frac{\left(\begin{array}{l}
l \\
i
\end{array}\right)\left(\begin{array}{l}
l \\
j
\end{array}\right)}{\left(\begin{array}{c}
n \\
j-l
\end{array}\right)},
$$

which is indeed an identity, since both sides equal

$$
\frac{j !(n+l-j) !}{i ! n !(l-i) !} .
$$

This concludes the proof of the Theorem.

Q.E.D.

As an immediate consequence we obtain the following corollary:

5.7 Corollary: The most general equation for the evolution of curves on $\mathbf{R P}^{n-1}$ which is invariant under the projective action of $\operatorname{SL}(n, \mathbf{R})$ is given by

$$
\phi_{t}=\Phi(\mathrm{Id}+A) \mathscr{T},
$$

where $\Phi$ and $A$ are given by (5.5) and (5.6), and $\mathscr{T}$ is any vector differential invariant for the action.

\section{THE EQUIVALENCE OF EVOLUTIONS}

The SL $(2, \mathbf{R})$ case. We will describe the case $n=2$ first to illustrate the procedure to be followed in general. In this case $\mathscr{b}_{n} \equiv \mathscr{b}_{2}$ is the manifold of Hill's operators of the form

$$
\frac{d^{2}}{d \theta^{2}}+u
$$

and $\mathscr{C}_{n} \equiv \mathscr{C}_{2}$ is the space of curves on the projective line such that $d \phi / d \theta=\phi_{\theta} \neq 0$. By Theorem 4.3 , the most general evolution on $\mathscr{C}_{2}$ invariant under the $\operatorname{SL}(2, \mathbf{R})$ action is given by the equation

$$
\phi_{t}=\phi_{\theta} \mathscr{T} .
$$

Here $\mathscr{T}$ is a differential invariant of the action, that is, a function of $S(\phi)$ and its derivatives with respect to $\theta$, where $S(\phi)$ is the Schwartzian derivative of $\phi$ given by (4.9).

Given a curve $\phi$ on $\mathscr{C}_{2}$ with a monodromy $M$, there is a unique operator of the form (6.1) such that $\xi=\left(\xi_{1}, \xi_{2}\right)=\left(\phi^{\prime-1 / 2}, \phi^{\prime-1 / 2} \phi\right)$ is its solution curve. Once the solution curve is fixed one can factor $L=(\partial-v)(\partial+v)$ in a unique fashion so that $(\partial+v) \xi_{1}=0$ and $(\partial-v)(\partial+v) \xi_{2}=0$. More precisely,

$$
v=-\frac{\left(\phi^{\prime-1 / 2}\right)^{\prime}}{\phi^{\prime-1 / 2}}=\frac{1}{2} \frac{\phi^{\prime \prime}}{\phi^{\prime}} .
$$


Assume now that $\phi$ is evolving according to equation (6.2). Then, due to its dependence on $\phi, v$ will be evolving following the equation:

$$
\begin{aligned}
v_{t} & =\frac{D v}{D \phi}\left(\phi^{\prime} \mathscr{T}\right)=-\frac{D\left(\left(\phi^{\prime-1 / 2}\right)^{\prime} / \phi^{\prime-1 / 2}\right)}{D \phi}\left(\phi^{\prime} \mathscr{T}\right) \\
& =-\partial\left(\frac{1}{\phi^{\prime-1 / 2}} \frac{D\left(\phi^{\prime-1 / 2}\right)}{D \phi}\right)\left(\phi^{\prime} \mathscr{T}\right) \\
& =\frac{1}{2} \partial\left(\frac{1}{\phi^{\prime}} \partial\right)\left(\phi^{\prime} \mathscr{T}\right)=\frac{1}{2} \partial(\partial+2 v) \mathscr{T} .
\end{aligned}
$$

On the other hand, the evolution of $v$ according to the Kupershmidt-Wilson definition is given by

$$
v_{t}=-\frac{1}{2} \partial \frac{\delta \mathscr{H}}{\delta v}
$$

for some Hamiltonian functional $\mathscr{H}$ depending on $v$ and its derivatives.

Two comments are due at this point. First of all, let $\delta \mathscr{H} / \delta v$ be the variational derivative of $\mathscr{H}$ with respect to $v$, and let $\widehat{\delta \mathscr{H} /} \delta u$ denote the corresponding variational derivative with respect to the variables $u$, expressed in terms of $v$. Then the following equality holds:

$$
\frac{\delta \mathscr{H}}{\delta v}=\left(\frac{D u}{D v}\right) * \frac{\widehat{\delta \mathscr{H}}}{\delta u} .
$$

The proof of this statement can be found in Ref. 3, p. 420.

The second comment is as follows: notice that $\delta \mathscr{H} / \delta u$ is a differential invariant, since it depends on the coefficients $u$ and their derivatives, which are themselves independent differential invariants. This was pointed out throughout Sections III and IV. On the other hand, the latter result doesn't hold for the Fréchet derivative with respect to $v$, since the coefficients of the first-order factors are not invariant with respect to the action of $\operatorname{SL}(n, \mathbf{R})$. Thus, in order to find the equivalence of evolutions, we must write the Adler-Gel'fand-Dikii evolution of $v$ in terms of the Hamiltonian as a function of $u$. That is, the proper correspondence is between the $\phi$-evolution and the $u$-evolution, since the coefficients $u$ are invariants of the $\operatorname{SL}(n, \mathbf{R})$ action. We are using the variables $v$ to simplify calculations, since the original definition of the Adler-Gel'fand-Dikii bracket in terms of the $u$ coordinates is too complicated. These two comments are obviously valid in the general case and not only for $n=2$.

Returning to (6.3), we can rewrite this equation as

$$
v_{t}=-\frac{1}{2} \partial\left(\frac{D u}{D v}\right) * \frac{\widehat{\delta \mathscr{H}}}{\delta u}
$$

But $u=-v^{2}+v^{\prime}$ in this case, so that $(D u / D v)^{*}=-(\partial+2 v)$, and we have thus shown that the evolution due to the dependence of $v$ on $\phi$ is identical to the Adler-Gel'fand-Dikii evolution provided that

$$
\mathscr{T}=\frac{\delta \mathscr{H}}{\delta u}
$$

By Helmholtz's theorem, an SL $(n, \mathbf{R})$-invariant evolution (6.2) is associated to an AdlerGel'fand-Dikii Hamiltonian evolution (6.3) if and only if its differential invariant $\mathscr{T}$ has selfadjoint Fréchet derivative.

The general case. The proof for other values of $n$ follows the same ideas that we showed in the case $n=2$. The main practical problem is, of course, the complication of the calculations involved. Our goal is to show that whenever a non-degenerate right-hand oriented projective curve 
$\phi$ follows the evolution $\phi_{t}=\Phi(\operatorname{Id}+A) \mathscr{T}$, then the corresponding coefficients of its associated operator follow the Adler-Gel'fand-Dikii evolution provided the vector differential invariant $\mathscr{T}$ is related to $\delta \mathscr{G} / \delta u$ in a suitable way. For this to be the case, $\mathscr{T}$ must satisfy a condition coming from Helmholtz's theorem analogous to the one at the end of the last section. In this section we will simplify the problem and establish a closer connection between both evolutions before explaining where the main problem lies. In any case, using this simplified version it is relatively easy to establish the equivalence of both evolutions for a fixed value of $n$.

6.1 Proposition: A choice of modified variables $v$ can be expressed in terms of the basic homogeneous variables as $v=\Omega^{-1} y$, where

$$
y_{0}=\frac{1}{n} q_{n}^{n-1}, \quad y_{i}=q_{i}^{i-1}-q_{i+1}^{i}+\frac{1}{n} q_{n}^{n-1}, \quad 1 \leqslant i \leqslant n-2,
$$

$q_{1}^{0}=0$ by definition, and $\Omega$ is the Vandermonde matrix defined by

$$
\Omega=\left(\begin{array}{cccc}
1 & 1 & \ldots & 1 \\
\omega & \omega^{2} & \ldots & \omega^{n-1} \\
\vdots & \vdots & \ddots & \vdots \\
\omega^{n-2} & \omega^{2(n-2)} & \ldots & \omega^{(n-1)(n-2)}
\end{array}\right)
$$

Proof: It suffices to show that the we can factor $L=\partial^{n}+u_{n-2} \partial^{n-2}+\cdots+u_{1} \partial+u_{0}$ $=\left(\partial+y_{n-1}\right) \cdots\left(\partial+y_{0}\right)$ uniquely so that the coefficients $y$ are given by (6.4). Let us lift $\phi$ to a solution of $L$. The solution is given uniquely by $\xi=\left(\xi_{1}, \xi_{2}, \ldots, \xi_{n}\right)=W_{n-1}^{-1 / n}\left(1, \phi_{1}, \phi_{2}, \ldots, \phi_{n-1}\right)$. We choose $y$ so that $\xi_{i}$ is a solution of

$$
\left(\partial+y_{i-1}\right) \cdots\left(\partial+y_{1}\right)\left(\partial+y_{0}\right) \xi_{i}=0, \quad i=1, \ldots, n .
$$

It is not hard to show that there is a unique choice for $y$, namely,

$$
y_{i}=\frac{\omega_{i-1}^{\prime}}{\omega_{i-1}}-\frac{\omega_{i}^{\prime}}{\omega_{i}}, \quad i=1,2, \ldots, n,
$$

where $\omega_{i}=W\left(\xi_{1}, \ldots, \xi_{i+1}\right)$. Indeed, notice that $y_{0}+y_{1}+\cdots+y_{i-1}$ is the coefficient of $\partial^{i-1}$ in $\left(\partial+y_{i-1}\right) \cdots\left(\partial+y_{1}\right)\left(\partial+y_{0}\right)$. On the other hand, if $\xi_{1}, \ldots, \xi_{i}$ are the independent solutions of this operator, then the coefficient of $\partial^{i-1}$ is given by $-\omega_{i-1}^{\prime} / \omega_{i-1}$, cf. (2.3), from which (6.5) easily follows. From the form of $\xi$ we get that $\omega_{i}=W_{i} / W_{n-1}^{(i+1) / n}$. Substituting in (6.5) we get (6.4) for $i \geqslant 1$ straightforwardly. The formula for $y_{0}$ is an immediate consequence of the equation $\left(\partial+y_{0}\right) \xi_{1}=\left(\partial+y_{0}\right) W_{n-1}^{-1 / n}=0$, while the relationship $v=\Omega^{-1} y$ is simply the definition (3.2) of $v$.

We want to see next under what conditions the evolution of $v$

Q.E.D.

$$
v_{t}=\frac{D v}{D \phi} \phi_{t}=\frac{D v}{D \phi} \Phi(\operatorname{Id}+A) \mathscr{T}
$$

induced by the $\operatorname{SL}(n, \mathbf{R})$-invariant evolution of $\phi$ coincides with the Adler-Gel'fand-Dikii Hamiltonian evolution

$$
v_{t}=-\frac{1}{n} \partial J\left(\frac{D u}{D v}\right)^{*} \frac{\widehat{\delta \mathscr{H}}}{\delta u}
$$


where $\widehat{\delta \mathscr{H}} \mid \delta u$ is defined as in the case $n=2$. We are going to simplify both equations before proceeding with further calculations. Using the previous proposition, we can write (6.6) as

$$
v_{t}=\Omega^{-1} S \frac{D q}{D \phi} \Phi(\operatorname{Id}+A) \mathscr{T},
$$

where

$$
\left.S=\mid \begin{array}{cccccc}
0 & 0 & 0 & \ldots & 0 & 1 / n \\
-1 & 0 & 0 & \ldots & 0 & 1 / n \\
1 & -1 & 0 & \ldots & 0 & 1 / n \\
0 & \ddots & \ddots & \ddots & \vdots & \vdots \\
\vdots & \ddots & 1 & -1 & 0 & 1 / n \\
0 & \ldots & 0 & 1 & -1 & 1 / n
\end{array}\right)
$$

and $\quad q=\left(q_{k}^{k-1}\right)_{k=2}^{n}$. Since $\quad q_{k}^{k-1}=W_{k-1}^{\prime} / W_{k-1}$, we have $D q_{k}^{k-1} / D \phi=\partial\left(\left(1 / W_{k-1}\right)\right.$ $\left.\left(D W_{k-1} / D \phi\right)\right)$. Thus, the equality of the evolutions (6.6) and (6.7) will be proved once we show that

$$
\Omega^{1} S\left(\frac{1}{W} \frac{D W}{D \phi}\right) \Phi(\mathrm{Id}+A) \mathscr{T}=-\frac{1}{n} J\left(\frac{D u}{D v}\right) * \widehat{\delta \mathscr{H}}
$$

where by $(1 / W)(D W / D \phi)$ we mean the matrix whose $(i, j)$ entry is given by $\left(1 / W_{i}\right)\left(D W_{i} / D \phi_{j}\right)$. Straight-forward multiplication of matrices shows that (6.8) becomes

$$
\frac{1}{W} \frac{D W}{D \phi} \Phi(\operatorname{Id}+A) \mathscr{T}=R\left(\frac{D u}{D y}\right) * \widehat{\delta \mathscr{H}}
$$

where

$$
R=-\frac{1}{n} S^{-1} \Omega J \Omega^{t}=\left(\begin{array}{cccccc}
-1 & 1 & 0 & 0 & \ldots & 0 \\
-2 & 1 & 1 & 0 & \ldots & 0 \\
-3 & 1 & 1 & 1 & \ldots & 0 \\
\vdots & \vdots & \ddots & \ddots & \ddots & \vdots \\
-(n-2) & 1 & \ldots & 1 & 1 & 1 \\
-(n-1) & 1 & \ldots & 1 & 1 & 1
\end{array}\right)
$$

We conjecture (6.9) to be true whenever

$$
\frac{\delta \mathscr{H}}{\delta u}=T \mathscr{C} \mathscr{T},
$$

where 


$$
T=\left(\begin{array}{ccccccc}
0 & \ldots & 0 & 0 & 0 & 0 & 1 \\
0 & \ldots & 0 & 0 & 0 & 1 & \partial \\
0 & \ldots & 0 & 0 & 1 & \left(\begin{array}{l}
2 \\
1
\end{array}\right) \partial & \partial^{2} \\
0 & \ldots & 0 & 1 & \left(\begin{array}{l}
3 \\
1
\end{array}\right) \partial & \left(\begin{array}{c}
3 \\
2
\end{array}\right) \partial^{2} & \partial^{3} \\
\vdots & . & . & . & . & . & \vdots \\
0 & 1 & \left(\begin{array}{c}
n-3 \\
1
\end{array}\right) \partial & \left(\begin{array}{c}
n-3 \\
2
\end{array}\right) \partial^{2} & \left(\begin{array}{c}
n-3 \\
3
\end{array}\right) \partial^{3} & \ldots & \partial^{n-3} \\
1 & \left(\begin{array}{c}
n-2 \\
1
\end{array}\right) \partial\left(\begin{array}{c}
n-2 \\
2
\end{array}\right) \partial^{2} & \left(\begin{array}{c}
n-2 \\
3
\end{array}\right) \partial^{3} & \ldots & \left(\begin{array}{c}
n-2 \\
n-3
\end{array}\right) \partial^{n-3} & \partial^{n-2}
\end{array}\right)
$$

and $\mathscr{C}$ is a certain upper triangular matrix of the form

$$
\mathscr{L}=\left(\begin{array}{cccccc}
1 & 0 & m_{1}^{1} & m_{1}^{2} & \ldots & m_{1}^{n-3} \\
0 & 1 & 0 & m_{2}^{2} & \ldots & m_{2}^{n-3} \\
\vdots & \ddots & \ddots & \ddots & \ddots & \vdots \\
0 & \ldots & 0 & 1 & 0 & m_{n-3}^{n-3} \\
0 & \ldots & 0 & 0 & 1 & 0 \\
0 & \ldots & 0 & 0 & 0 & 1
\end{array}\right),
$$

whose matrix elements $m_{i}^{j}$ are all functions of the coefficients $u_{i}$ and their derivatives. On the other hand, if $H_{u}=\sum_{k=1}^{n} h_{k} \partial^{-k}$, the vector $\left(h_{1}, \ldots, h_{n-1}\right)$ is easily seen to be related to the gradient of $\mathscr{H}$ through the matrix $T$, exactly the same way $\mathscr{C} \mathscr{T}$ is. (The coefficient $h_{n}$ of $H_{u}$ is determined by the other coefficients, from the condition that the associated Hamiltonian vector field $V_{H_{u}}$ be tangent to $\mathscr{b}_{n}$.) That is, (6.9) will hold provided that a certain linear combination of $\mathscr{T}$ with differential invariant coefficients coincides with the coefficients $\left(h_{1}, \ldots, h_{n-1}\right)$ of the pseudodifferential operator $H_{u}$ defining the evolution of $u$. As in the previous section, the necessary and

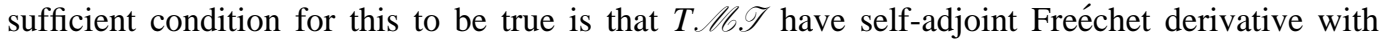
respect to $u$.

One can see this relation between $\mathscr{T}$ and $H_{u}$ from a different point of view. Any relative invariant is the product of the particular solution $\mu$ of (4.8) given by (5.4), times an invertible matrix of differential invariants, such as $\mathscr{L}$. That is, we conjecture that one can find a relative invariant of the form $\tilde{\mu}=\Phi(I d+A) \mathscr{C}^{-1}$ such that the invariant evolution $\phi_{t}=\tilde{\mu} \mathscr{T}$ is equivalent to the Adler-Gel'fand-Dikii evolution whenever $T \mathscr{T}$ has self-adjoint Fréchet derivative with respect to $u$, so that $\mathscr{T}$ is equal to the coefficients of $H_{u}$ for a certain Hamiltonian pseudo-differential operator $H$. This gives a Hamiltonian interpretation of $\mathfrak{s l}(n, \mathbf{R})$ differential invariants satisfying the above integrability condition.

There exists an ansatz for the explicit expression of $\mathscr{A} b$ which proves the conjecture up to $n=6$. For $n=2$ and $3 \mathscr{C} 6$ is the identity. For $n=4$

$$
\mathscr{L}=\left(\begin{array}{ccc}
1 & 0 & -\frac{1}{2} u_{2} \\
0 & 1 & 0 \\
0 & 0 & 1
\end{array}\right),
$$

and for $n=5$ 


$$
\mathscr{C b}=\left(\begin{array}{cccc}
1 & 0 & -\frac{7}{10} u_{3} & \frac{3}{5} u_{2}-u_{3}^{\prime} \\
0 & 1 & 0 & -\frac{2}{5} u_{3} \\
0 & 0 & 1 & 0 \\
0 & 0 & 0 & 1
\end{array}\right) .
$$

But for higher dimensions $\mathscr{L} b$ involves more complicated expressions of the coefficients $u$ which can be explicitly generated by induction.

Finally, (6.9) becomes the following equality of matrices:

$$
\frac{1}{W} \frac{D W}{D \phi} \Phi(\operatorname{Id}+A)=R\left(\frac{D u}{D y}\right)^{*} T \mathscr{L l} \text {. }
$$

Let us analyze this equation. The matrix $(1 / W)(D W / D \phi) \Phi$ is easily calculated to have as $(i, j)$ entry the expression $\sum_{r=1}^{i} \Sigma_{s=0}^{r}\left(\begin{array}{l}r \\ s\end{array}\right) q_{1 \ldots r+j-s \ldots i} \partial^{s}$, where $r+j-s$ is in the $r$ th place. Thus, the left-hand side of (6.10) does not represent a major problem. With respect to the right-hand side, we can write this expression in terms of $q$ 's. There are old formulas ${ }^{14}$ relating $u$ 's to $q$ 's which, in our notation, become

$$
u_{m}=\sum_{i=0}^{n-m}(-1)^{n-m-i}\left(\begin{array}{c}
m+i \\
i
\end{array}\right) \Lambda_{i} q_{n}^{m+i}, \quad 0 \leqslant m \leqslant n-2,
$$

where $q_{n}^{0}=0$ by definition, $\Lambda_{0}=1, \Lambda_{1}=(1 / n) q_{n}^{n-1}$, and $\Lambda_{i}$ is given by the following recurrent formula:

$$
\Lambda_{i}=\sum_{k=0}^{i-1}\left(\begin{array}{c}
i-1 \\
k
\end{array}\right) \Lambda_{k}\left(\Lambda_{1}\right)^{(i-1-k)}
$$

In particular, observe that the $\Lambda_{i}$ 's are all functions of $q_{n}^{n-1}$ and its derivatives. Using formulas similar to these and Lemma 5.3 skillfully enough, one should expect to be able (although this is by no means trivial) to simplify that part of the equation also. The main trouble lies on the choice of variables; notice how it would be very difficult to explicitly write both sides of the equation in terms of basic homogeneous variables and their derivatives, even after trying to use Lemma 4.3 and similar expressions. Also, since all the homogeneous expressions $q_{n}^{k}$ have very involved relationships with each other, the chances of using another obvious group of variables among them in an effective way are very small. The goal would be to find a different set of variables making the equivalence between the Adler-Ge'lfand-Dikii and the $\operatorname{SL}(n, \mathbf{R})$-invariant evolutions totally transparent.

\section{ACKNOWLEDGMENTS}

A.G-L. and R.H.H. would like to acknowledge the partial financial support of the DGES under Grant No. PB95-0401.

\footnotetext{
${ }^{1}$ M. Adler, "On a trace functional for formal pseudo-differential operators and the symplectic structure of the KdV," Invent. Math. 50, 219-48 (1979).

${ }^{2}$ I. M. Gel'fand and L. A. Dikii, "A family of Hamiltonian structures connected with integrable nonlinear differential equations," in I. M. Gel'fand, Collected Papers, Vol. 1 (Springer, New York, 1987).

${ }^{3}$ B. A. Kupershmidt and G. Wilson, "Modifying Lax equations and the second Hamiltonian structure," Invent. Math. 62, 403-36 (1981).

${ }^{4}$ V. G. Drinfel'd and V. V. Sokolov, "Lie algebras and equations of KdV type," J. Sov. Math. 30, 1975-2036 (1985).

${ }^{5}$ P. J. Olver, G. Sapiro, and A. Tannenbaum, "Differential invariant signatures and flows in computer vision: A symmetry
} 
group approach," in Geometry-Driven Diffusion in Computer Vision, edited by B. M. ter Haar Romeny (Kluwer Academic, Dordrecht, 1994).

${ }^{6}$ P. J. Olver, Equivalence, Invariance and Symmetry (Cambridge University Press, Cambridge, 1995).

${ }^{7}$ G. Wilson, "On the antiplectic pair connected with the Adler-Gel'fand-Dikii bracket," Nonlinearity 5, 109-31 (1992).

${ }^{8} \mathrm{I}$. McIntosh, "SL $(n+1)$-invariant equations which reduce to equations of Korteweg-de Vries type," Proc. R. Soc. Edinburgh Sect. A 115, 367-81 (1990).

${ }^{9} \mathrm{~V}$. Yu. Ovsienko and B. A. Khesin, "Symplectic leaves of the Gel'fand-Dikii brackets and homotopy classes of non-degenerate curves,' Funct. Anal. Appl. 24, n. 1, 33-40 (1990).

${ }^{10}$ P. J. Olver, Applications of Lie Groups to Differential Equations (Springer, New York, 1993), 2nd ed.

${ }^{11}$ G.-H. Halphen, "Sur les invariants différentiels," in Euvres, Vol. 2 (Gauthier-Villars, Paris, 1913).

${ }^{12}$ S. Lie, "Uber Differentialinvarianten," in Gesammelte Abhandlungen, Vol. 6 (B. G. Teubner, Leipzig, 1927); see M. Ackerman and R. Hermann, Sophus Lie's 1884 Differential Invariant Paper (Math Sci Press, Brookline, Mass., 1975) for an English translation.

${ }^{13}$ A. Tresse, "Sur les invariants différentiels des groupes continus de transformations," Acta Math. 18, 1-88 (1894).

${ }^{14}$ E. J. Wilczynski, Projective Differential Geometry of Curves and Ruled Surfaces (B. G. Teubner, Leipzig, 1906). 
Journal of Mathematical Physics is copyrighted by AIP Publishing LLC (AIP). Reuse of AIP content is subject to the terms at: http://scitation.aip.org/termsconditions. For more information, see http://publishing.aip.org/authors/rights-and-permissions. 